\title{
Design and Implementation of an Identification System in Construction Site Safety for Proactive Accident Prevention
}

\author{
Huanjia Yang ${ }^{\mathrm{a}, *}$, David A.S. CHEW ${ }^{\mathrm{b}}$, Weiwei $\mathrm{Wu}^{\mathrm{c}}$, Zhipeng ZHOU ${ }^{\mathrm{c}}$, Qiming LI $^{\mathrm{c}}$ \\ ${ }^{a}$ Computer Science Department, FK Holywell Park, Loughborough University, Loughborough, LE11 3TU, United \\ Kingdom \\ b School of Civil and Environmental Engineering, Nanyang Technological University, Block N1, Level 1, Rm \\ N1-01b-48, 50 Nanyang Avenue, 639798, Singapore \\ ${ }^{\mathrm{c}}$ Dept. of Construction Management and Real Estate, Southeast University, No. 2, Si Pai Lou, Nanjing, 210096, \\ China \\ * Corresponding Author. Computer Science Department, FK Holywell Park, Loughborough University, \\ Loughborough, LE11 3TU, United Kingdom. Tel.: +44(0)7515 363547; fax: +44(0) 1509211586. \\ E-mail address: huanjiayang@hotmail.com (H. Yang).
}

\begin{abstract}
Identifying accident precursors using real-time identity information has great potential to improve safety performance in construction industry, which is still suffering from day to day records of accident fatality and injury. Based on the requirements analysis for identifying precursor and the discussion of enabling technology solutions for acquiring and sharing real-time automatic identification information on construction site, this paper proposes an identification system design for proactive accident prevention to improve construction site safety. Firstly, a case study is conducted to analyze the automatic identification requirements for identifying accident precursors in construction site. Results show that it mainly consists of three aspects, namely access control, training and inspection information and operation authority. The system is then designed to fulfill these requirements based on ZigBee enabled Wireless Sensor Network (WSN), Radio Frequency Identification (RFID) technology and an integrated ZigBee RFID sensor network structure. At the same time, an information database is also designed and implemented, which includes 15 tables, 54 queries and several reports and forms. In the end, a demonstration system based on the proposed system design is developed as a proof of concept prototype. The contributions of this study include the requirement analysis and technical design of a real-time identity information tracking solution for proactive accident prevention on construction sites. The technical solution proposed in this paper has a significant importance in improving safety performance on construction sites. Moreover, this study can serve as a reference design for future system integrations where more functions, such as environment monitoring and location tracking,
\end{abstract}


can be added.

Keywords: precursor; construction site; proactive prevention; Radio Frequency Identification; Wireless Sensor Network.

\section{Introduction}

Construction's safety record is unenviable, as the fatality and injury records in construction continue to plague the industry across the world (Gibb et al., 2006; Aneziris et al., 2008; Manu et al., 2010). In order to prevent fatal and injury accidents on construction sites, various existing research have been carried out to identify and analyze the causes of safety hazards and risks from an integral perspective of accidents (Cheng et al., 2010; Aneziris et al., 2010). On the other hand, there were also studies that concentrate on the causes of specific safety hazards, such as study on falling accidents (Goh et al., 2010), human-machine conflictions (Teizer et al., 2010a), electrical fatalities (Chi et al., 2009), steel erection fatalities (Beavers et al., 2009) and 'struck-by' injuries (Hinze et al., 2005). Among those studies, the accidents of falling from height on construction sites is the most commonly identified safety hazard (Chi et al., 2005; Aneziris et al., 2008; Saurin and Guimaraes, 2008).

It has been widely accepted that accidents are just the tip of an iceberg and henceforth understanding the cause of the accidents is of great significance for accident prevention. However, simply learning from the accidents that have already happened is not enough. For each particular incident, it is not acceptable to identify and record a specific hazard only after a related accident has already happened. It is therefore useful to study the near-miss accidents, which are deemed as another effective learning source and tracking method for accident prevention. Near-miss accidents were usually referred to as precursors of accidents (Bier and Mosleh, 1990), indicators of potential accidents (Brazier, 1994) or imminent signals of accidents (Jones et al., 1999). Phimister and Bier (2004) identified a near-miss accident as a special kind of precursor and defined it as an event in which no damages or injuries actually occurred but, under slightly different circumstances, could have resulted in great harm. In construction, the modified statistical triangle of accident causation described the same process from near-miss accidents to fatal accidents (Carter and Smith 2006). Cambraia et al. (2010) adopted the concept of a near-miss 
accident as an instantaneous event that involved the sudden release of energy and had the potential to generate an accident.

Many organizations have attempted to develop programs to identify and benefit from accident precursors, which were defined as the conditions, events and sequences that preceded and led up to accidents (Phimister and Bier, 2004). Similarly, Suraji et al. (2001) explained this concept as an undesired event, which was an unwanted incident immediately preceding and leading to an accident that did, or could have caused injury to construction personnel or the general public, or damage to property or the environment. When adopting a broad definition of precursor, it is not difficult to find that a near-miss accident is an important kind of precursor (Jones et al., 1999; Phimister and Bier, 2004). However, some organizations have chosen to limit the use of the term 'precursors' to near-miss accidents that exceed a specified level of severity (Phimister and Bier, 2004). Similarly, the objective of this research has also confined precursors to events that exceed a specified level of severity. It should be pointed out that a near-miss accident is identified in a much wider range of severities and may include all defects and abnormal events.

Though focusing on precursors and near miss-accidents on construction sites has been recognized as one of the important ways to improve safety on construction sites, its potentials have not been fully realized (Wu et al., 2010a). Recent research outcomes have shown that the information of identity about on-site staff, machine and material is highly related to accident precursors and near-miss accidents on construction sites (Wu et al., 2010b). In the meantime, investigation by $\mathrm{Lu}$ et al. (2010) also reveals that information which is crucial for safety management, such as labors' IDs, photos, access authorities, time of inspection, access control, personnel who conducts the checking, conditions of the machines, repair work done and so on. Such information can be handled by radio frequency identification (RFID) based automatic technologies. RFID refers to a branch of automatic identification technologies in which radio frequencies are used to capture and transmit data from a tag, or transponder (Ergen and Akinci, 2007). The potentials and benefits of applying RFID technology in construction site safety have been given much attention but the construction industry is slow in adopting the RFID technology in spite of its great potential (Wu et al., 2010b; Lu et al., 2010).

The development of numerous emerging information technologies has prompted research into their application to construction. Compared to other automatic identification systems, RFID is the 
most promising technology that does not require line-of-sight communication between tags and readers. Besides, RFID has inexpensive yet reliable tags, which provide more comprehensive tagging information and fast-speed reading. Chae and Yoshida (2010) applied RFID technology to the prevention of collision accidents with heavy equipment such as hydraulic excavators and cranes. Teizer et al. (2010b) introduced radio frequency remote sensing and actuating technology to improve construction safety, warning or alerting workers-on-foot and equipment operators in a proactive real-time mode when the equipment comes close to other objects or equipment. Navon and Berkovich (2005) have developed a model based on automatic, or semiautomatic, data collection for materials management and control. Navon and Sacks (2007) discussed the monitoring of automated data collection needs and the potential technologies to satisfy them. Ergen and Akinci (2007) highlighted the potential of RFID technology in enabling automated tracking of components/materials in dynamic and uncontrolled environments. WSN is a self-organized wireless network consisting of a large number of sensor nodes that interact with the physical world (Li and Liu 2007). Compared to other existing networking technologies, WSN is a low-power, low-cost, reliable and easy-to-implement wireless technology. It can accommodate a large number of nodes and can be deployed in a complex environment readily. In the construction industry, WSN has been applied in structural health monitoring (Sazonov 2004; Paek et al. 2005; Li and Liu 2007). Furthermore, Jang and Skibniewski (2009) created a framework for integrating the latest innovations in wireless sensor networks that automate tracking and monitoring construction assets using a combination of radio frequency and ultrasound signals.

One way to achieve RFID based applications for construction site safety is to implement the proposed systems proposed by Lu et al. (2010). Nevertheless, little research has been done in both the integrated analysis of automatic identification requirement and its technological solutions based on real time information on construction sites. Similar lack of research also applies to the investigation of the integration of RFID technology for automatic tracking of identity information in construction safety. In this paper, the integration of RFID system in sensor network architecture is investigated to enable an autonomous identification system in construction site safety for proactive accident prevention. Thus the objective of this paper is to address this challenge by designing and implementing an identification system in construction site safety for proactive accident prevention. This study will provide the technical solution, which is based on a dedicated 
RFID sensor network system, for tracking identity information for proactive accident prevention. It is therefore of great significance and importance in improving the safety performance on construction sites.

The paper is organized as follows: firstly, following the detailed background introduction, we briefly describe our case study methodology and the main technologies involved in our system design. The case study is then carried out with key information required for tracking near-miss accidents on construction sites that are being identified. Based on this outcome, the system is then designed, with system network architecture, database structure and implementation illustration being presented. A demonstration system is designed and tested to show the feasibility of our system design. Finally, a discussion and conclusion of this work is given.

\section{Methodology and enabling technologies}

\subsection{Empirical methods of case study}

Darbra and Casal (2004) indicates that useful information can be provided by the historical analysis of the accidents about the most frequent accidents, their origins and causes. At the same time, this analysis can also provide rich information about the precursors of accidents (Wu et al., 2010a; Phimister and Bier, 2004). In order to conduct an integrated analysis of automatic identification requirement about the identity information regarding on-site staff, machine and material, the historical records in USA from 1995 to 2008 are analyzed. The cases used in this research come from the U.S. Department of Occupational Safety and Health Administrative (OSHA). They are in database form, created by the software Microsoft Access. Moreover, in order to focus on common and conventional types of construction sites, accident events are limited to residential, commercial building and manufacturing plant in the statistical analysis. In total, 4640 cases in residential, commercial building and manufacturing plant from 1995 to 2008 are analyzed.

\subsection{RFID and WSN technology}

\subsubsection{RFID}

The development of promising information technologies, such as RFID and WSN has prompted 
research into their application to construction. Automatic Identification (Auto-ID) technologies are a broad term of technologies that enable the machines to identify objects. The key for Auto-ID technologies is their automatic data capture ability. RFID is a type of Auto-ID technologies that uses radio waves to automatically identify people or objects. Compared to the other Auto-ID technologies the RFID provides a non-contact data transfer between the RFID tags and the reader/interrogators without the need for a strictly obstacle-free, line-of-sight reading. Some of the RFID tags allow the information carried to be rewritten which improves the flexibility of the technology. Moreover, multiple tags can be read simultaneously by a RFID reader to improve efficiency.

The basic components of a typical RFID system include: the transponder or the tag, which is a microchip in which a unique serial code is stored and transmitted when necessary via an antenna attached; the RFID reader/interrogator, which is used to retrieve/receive the identify from the tags; There are generally 3 types of RFID tags depending on the power source used, which are the active, passive and semi-passive/semi-active tags. Each has its own features and is suitable for certain types of applications. Among the various technologies, the passive RFID is the one that has become the main ID technology of interest in the past decade. Because of its simple, powerless, low cost and reliable tag design, the passive RFID technology has the potential to be adopted in the large scale applications in which a massive number of tags need to be deployed. For the RFID technology discussed in this paper, we focus on the adoption of passive RFID systems in tracking near-miss incidents on construction sites.

RFID technologies feature efficient and low cost solution for identifying and tracking of objects and personnel. They have been adopted in a wide range of applications, such as healthcare (Katz and Rice, 2009), manufacturing (Huang et al., 2007) and food industries (Kelepouris et al., 2007). RFID in construction applications is not a new idea. Lu et al. (2010) has summarized the research of RFID in construction. Most of the research focuses on using RFID in the construction supply chain (Wang et al., 2007), construction quality inspection and management (Wang, 2008) and construction assets tracking (Chae and Yoshida, 2010; Jang and Skibniewski, 2009; Goodrum et al., 2006).

\subsubsection{WSN and ZigBee}


WSN is a type of ad-hoc wireless network composed of a large number of spatially distributed autonomous sensor nodes that interact with the physical world for remote monitoring and control applications. The ZigBee technology, which is built based on the IEEE802.15.4 standard (Baronti et al., 2007), is one of the most popular WSN standards. Its specification is maintained by the ZigBee Alliance which consists of members from both academic and industry. The ZigBee technology features a self-organized and multi-hop wireless network structure, which provides an easy and fast system implementation. A typical ZigBee network structure is shown in Fig. 1. The IEEE 802.15.4 standard defines the Physical and MAC layers, in which two device types, the Full Function Devices (FFDs) and Reduced Function Devices (RFDs) are used to form a low energy wireless network with a star topology. ZigBee defines a network layer upon the IEEE 802.15.4 structure. It introduces coordinator, router and end device as the three main types of network level devices that construct a wireless sensor network with mesh topology. The ZigBee coordinator is based on IEEE802.15.4 FFD and is responsible for establishing the network, managing the joining of network devices and allocating network address blocks. A ZigBee network must have one and only one coordinator. The ZigBee router devices are also based on IEEE802.15.4 FFD. They construct the backbone of the ZigBee network and are responsible for the multi-hop data relay between network nodes. Both the ZigBee coordinator and the ZigBee routers can connect with multiple nodes in the ZigBee network so that a mesh network topology can be built. The ZigBee end devices are based on IEEE802.15.4 RFD. Each of them can connect to only one parent node, which must be either a coordinator or a router device. The end devices can be deemed as the leaf nodes of the network and can only be accessed through their parent nodes.

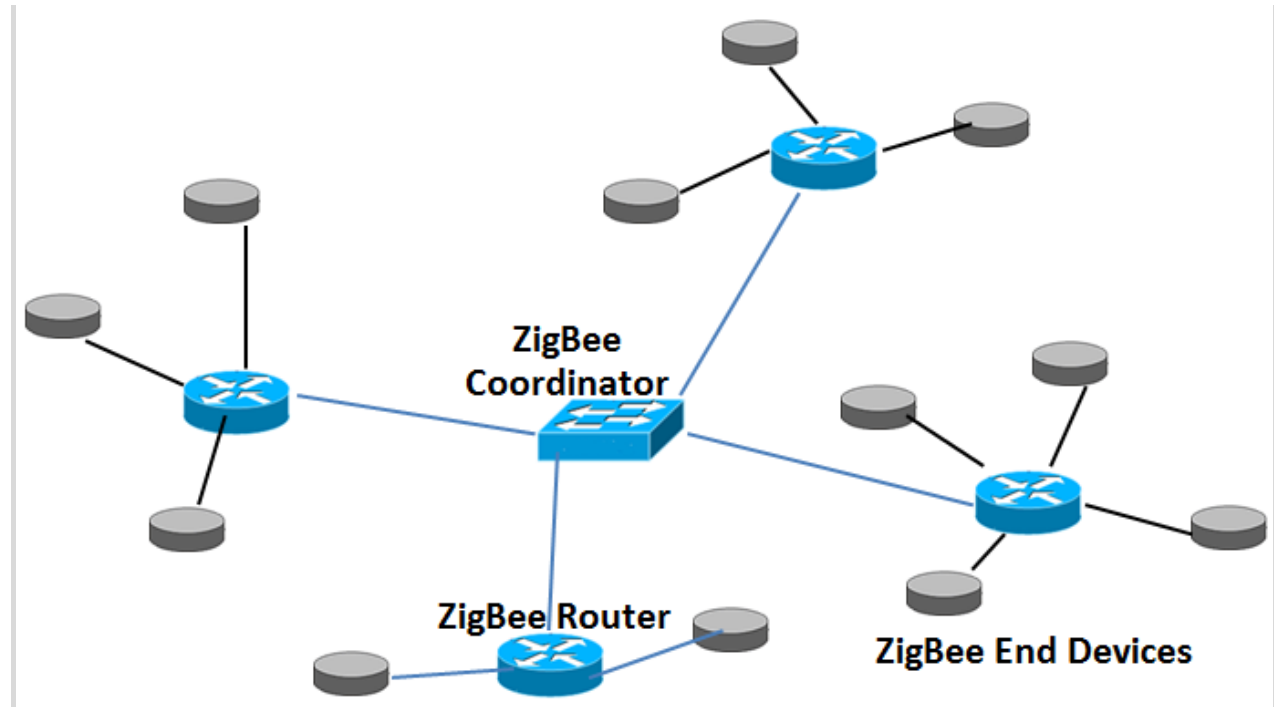


Fig. 1. Typical ZigBee Network Structure.

The ZigBee technology provides a reliable, low cost and easy to deploy wireless sensor network solution. In our research, we try to extend the application of ZigBee network systems. We consider that a ZigBee system not only transmits the sensory data from the end nodes deployed, but also has the potential of providing a wireless data transmission network structure from which the other types of information gathering devices, such as RFID readers, may also benefit. It has been recognized that there is a great potential in combining the ZigBee and RFID technologies to achieve a system that has the features from both sides. In our previous research, such an integrated system is called a ZigBee RFID Sensor Network. Various system architectures at network level have been studied for general integrations of RFID and WSN (Yang and Yang, 2007).

\section{Analysis and Results}

\subsection{Results of case study}

In total, 4640 cases are analyzed using the proposed methods of $\mathrm{Wu}$ et al. (2010a). The results show that the number of accidents that are related to identity information account for $77.8 \%$. Table 1 shows the results of major precursors related to identity information.

Table 1. Results of case studies that are related to identity.

\begin{tabular}{lll}
\hline \multicolumn{2}{c}{ Major near-miss accidents related to identity information } & Requirement Type \\
\hline 1 & Material is not tested for a long time. & Inspection Information \\
2 & Materials are not qualified. & Access Control and \\
& & Inspection Information \\
3 & Machine is not tested for a long time. & Inspection Information \\
4 & Poor experience and improper use of PPE. & Training Information \\
5 & Unauthorized to operate the equipment. & Operation Authority \\
6 & Workers do not operate in the right procedure. & Training Information \\
7 & Unauthorized to access hazard area. & Access Control
\end{tabular}


8 False procedure causes de-activation of electrical Access Control and Training systems. Information

9 Sloped sides, trench boxes or shoring are not operated in Training Information a right procedure.

From the results showing in Table 1, it can be concluded that automatic identification requirement in construction site safety mainly consists of three aspects: access control (on-site staff, material and machine), training and inspection information (on-site staff, material and machine) and operation authority (on-site staff and machine).

\subsection{Technical system development}

\subsubsection{ZigBee and RFID Sensor Network}

The system design is based on the ZigBee technology, UHF passive RFID technology and the ZigBee RFID sensor network architecture. The ZigBee technology is chosen as the WSN backbone because it provides a self-organized, low cost and easy to deploy network system structure that has the required flexibility for use as a plug-and-play system in construction site management applications with inconsistent environment. As a worldwide standard, the ZigBee devices from different manufacturers can work together which makes the system design more scalable. Passive UHF RFID system is currently the most popular RFID standard for item level identification. Regularized by EPCglobal and supported by many major companies and government agencies, such as Wal-Mart, US Department of Defense (DoD) and DHL, it is expected to be the unified auto-ID technology for item level tracking in the near future. A RFID Sensor Network is a combined technology that integrates RFID, wireless sensor networks and sensors into a unified all-in-one system, which features low cost, easy-to-implement, robust and highly flexible system structure. The various system architectures of RFID sensor network was investigated in our previous research (Yang and Yang, 2007), in which the "reader as a sensor" architecture is adopted in our system design.

\subsubsection{System architecture design}

The system design is fully based on the results of the application requirements analysis in the 
previous sections. Three primary types of RFID data terminal, namely the main access control reader, equipment access control reader and handheld reader terminal, are considered in the system integration. Fig. 2 shows the structure of our system design. The network backbone of the system is constructed by low-power wireless devices that are organized in a ZigBee wireless sensor network. The advantages of using a ZigBee WSN network backbone is that the system can benefit from a pure wireless backbone with simplified implementation procedure and improved flexibility during operation. This can be particularly important in a construction site where the physical environment is usually inconsistent during the system implementation and operation. Moreover, the system structure is divided into three layers: the ZigBee coordinator layer, the router layer and the end device layer.

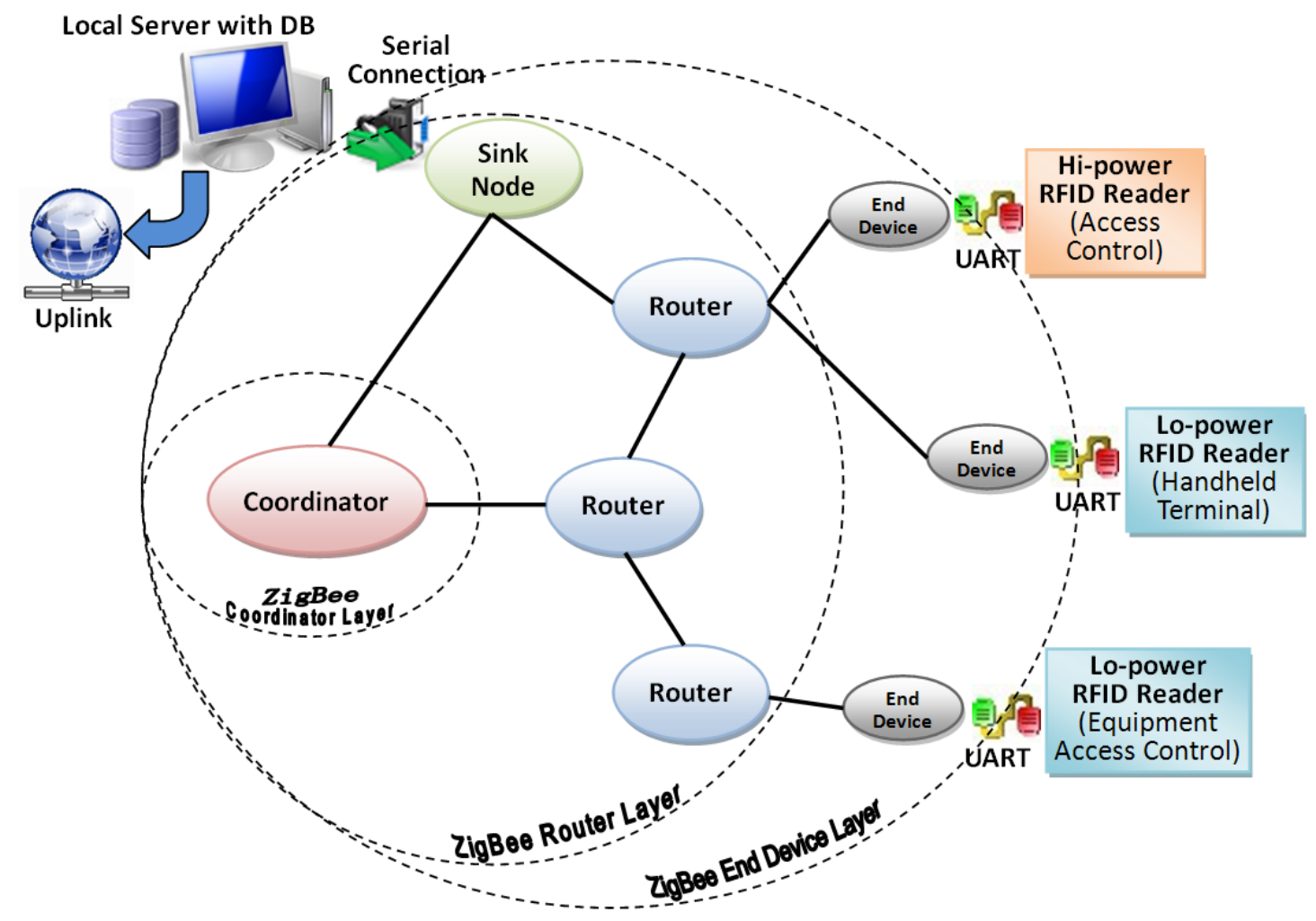

Fig. 2. System structure.

ZigBee coordinator layer: A ZigBee coordinator device, which is the only device located in the top layer of the network backbone, is essential in such a system design. It is responsible for establishing the ZigBee network, adding the routers that are connected directly to it into the network and allocating network address blocks to them. 
ZigBee router layer: After the network establishment, several ZigBee router devices can be activated and join the network. As the ZigBee devices are usually designed with low-power transmission profile, they are not guaranteed a direct connection among each other. The router devices are thus responsible for maintaining a mesh network backbone that fully covers the construction site, so that all the devices can communicate with each other via a multi-hop wireless path.

A fixed computing device, such as a desktop or a laptop that has a higher level computing ability comparing to the ZigBee network nodes, operates as the local server. It accommodates the local database service where all the functions that require either substantial data storage or high computing resource should be processed on the server. Those functions include the information databases (in section 3.3), the alarm or access control policies and the decision-making system. Furthermore, the network management and configuration should also be handled by the server through corresponding user interfaces. In addition, the server can also be connected to remote information centers via an uplink network connection, such as internet service or a private network, to report selected information for further enterprise integrations required.

In order to retrieve data from the RFID sensor network, the server is physically connected to a sink node which operates as a ZigBee router device and provides network access for the server. The RFID reading information as well as the inquiries from all the terminals is reported to the sink node. The sink node connects and communicates with the server computer via a serial cable connection. Actually, it is technically possible for the sink node to operate as an end device in the network. The reason why we prefer a router based sink node is that such a node will be a data aggregation point within the network. This means that it will be undertaking a relatively large amount of data transmission, especially for incoming data flow compared to the other network nodes. When the sink node operates as an end device in the network, it can only communicate with one router device that has been assigned as its direct parent node. As in a multi-hop mesh network this results in a high network traffic load on the parent router node, as all the incoming data packets to the sink node must be relayed by this particular router. The router devices are stand-alone devices with limited hardware and power resources. High traffic load on a particular network node will cause data packet congestion and long transmission delay. It will also shorten the device lifetime when the system operates without external power supply in certain 
circumstances. Furthermore, the system becomes vulnerable and less reliable because the router node becomes a "key point" node in the network which means that its failure will result in the whole system being out of function. For a demonstration system or a small-scale system such effects may not influence the system performance seriously, they are however critical in the systems that operate in large-scale implementations like a real construction site where hundreds of network nodes can be involved. Thus in the structure design of a wireless mesh network, we need to distribute the network traffic load as evenly as possible in order to improve system robustness and prolong the network lifetime, especially during extreme or unexpected situations. In this case, a router-based sink node is recommended because it can have multiple connections to other adjacent routers. The network traffic can thus be distributed and a failure of some of the routers will not immediately bring down the data service.

ZigBee end device layer: Located at the bottom level of the system architecture are the RFID readers which are data collectors. Reader devices are integrated with ZigBee compatible modules that operate as end devices in the system network. Similar to the sink node, the reader nodes are technically possible to operate either as routers or as end devices. However, in contrary to our conclusion for the sink node, the reader nodes are chosen to operate as end devices, for which the reasons are discussed next.

Firstly, the equipment/vehicle access control nodes and the handheld terminal nodes are mobile devices, which means their physical locations are subject to changing over the time. Those nodes will be operating on batteries and need to be power-efficient. As ZigBee routers participate in packet relaying within the network which means they not only need to conduct much more frequent RF transmission but also need to be active all the time. These functions significantly reduce the battery life and make the use of ZigBee routers as mobile nodes impractical in real applications. According to our experiment, a ZigBee router with two AAA size batteries can work on its own for only up to 24 hours before the battery is flat. This has not even taken into consideration of the power consumption of the RFID reader modules that have higher power consumption during their operation. On the other hand, ZigBee end devices are based on IEEE802.15.4 RFD (Reduced Function Device); that means they have a simpler structure, lower cost and less energy consumption because of their simple role in the network. They do not take part in the network routing mechanism and can thus be put into sleep-mode while idling. Using 
the same set of two AAA batteries the measurement and calculation on our hardware suggest that with a 0.5 second RF active time and 3 minute sleep time an end device can expect a battery life up to a year, which can be further extended to 2 years if the application can allow either a longer sleep time or a larger battery like two high capacity AA cells. This enables the mobile node to have a much reasonable power consumption level, thus making end device based reader nodes more suitable for our application.

In addition, another reason to favor end devices as mobile nodes is that the mobility of routers causes extreme routing overhead because their movement causes continuous changing of the logical topology in the network backbone and results in the mesh network constantly being reorganized (Sun et al., 2007). The movement of a router also results in all the end devices coupled with it being disconnected and having to search and join the network again and again, which is a very power-consuming operation. The movement of router devices in ZigBee network causes heavier traffic, inconsistent network topology and larger energy consumption not only by itself but also by all the end devices.

On the other hand, end devices are also preferred to be used as the fixed field access control nodes for two reasons. Firstly, they are more power-efficient and could function longer without external power support in certain circumstances. Secondly, increasing router number results in more hierarchies in network topology. According to the work of Liang et al. (2006), the more hierarchy a ZigBee network has, the more complexity it has with a concomitant lowering of efficiency in mobility support. Thus in summary, the end device based RFID reader nodes are more suitable for our application.

High-power RFID reader is used for the field access control, because in this case a fast reading speed is required as it is likely for the RFID reading to be performed with a relatively long tag-reader distance. According to our experiments with various readers, the low-power embedded reader modules tend to have a shorter reading range and a longer tag reading delay, thus a highpower RFID reader with better reading performance is preferred. On the other hand, the vehicle access control readers and the hand-held terminal readers do not have such strict criteria for reading performance. They can be positioned very close to the tags, and a much longer reading delay is acceptable. Taken into consideration that the device design for them focuses mainly on mobility and power consumption, the low-power embedded reader modules can be adopted to 
significantly reduce the device size and power consumption of those reader nodes. In order to achieve long reading distance and fast reading speed, while also taking into consideration that third party construction materials are likely to be tagged with UHF passive tags, the UHF passive RFID readers that are compatible with current UHF standards are the best choices for our application.

\subsection{Information database - the Identification System in Construction Site Safety (ISiCSS)}

The ISiCSS is designed in a user friendly way based on Microsoft Access 2007. In ISiCSS, tables, queries, reports and forms are designed based on Macro and Module. Buttons are provided in properly designed data rendering pages based on the Visual Basic for Application (VBA).

\subsubsection{Data organization}

Due to the limited memory and computing capability of RFID and WSN devices, a central information database is required for information storage and query. Based on the primary key information, such as ID number provided by RFID, the whole information set can be organized and queried for further analysis. In ISiCSS, the database is designed to consist of three parts of information including on-site staff, material and machine, as shown in Table 2.

Table 2. Fields of on-site staff, material and machine.

\begin{tabular}{lll}
\hline On-site staff & Material & Machine \\
\hline staff ID & material ID & equipment ID \\
nationality & production company & production company \\
sex & expiration date & expiration date \\
stature & by piece & type of equipment \\
type of company & volume & remark \\
education background & purchaser & maintenance staff \\
participate in similar work & checker & times repaired heavily \\
attitude to near-miss & remark & times undergoing near-miss \\
work certificate & test time & equipment name \\
perceptual competency & test company & production date \\
response competency & material name & using time
\end{tabular}




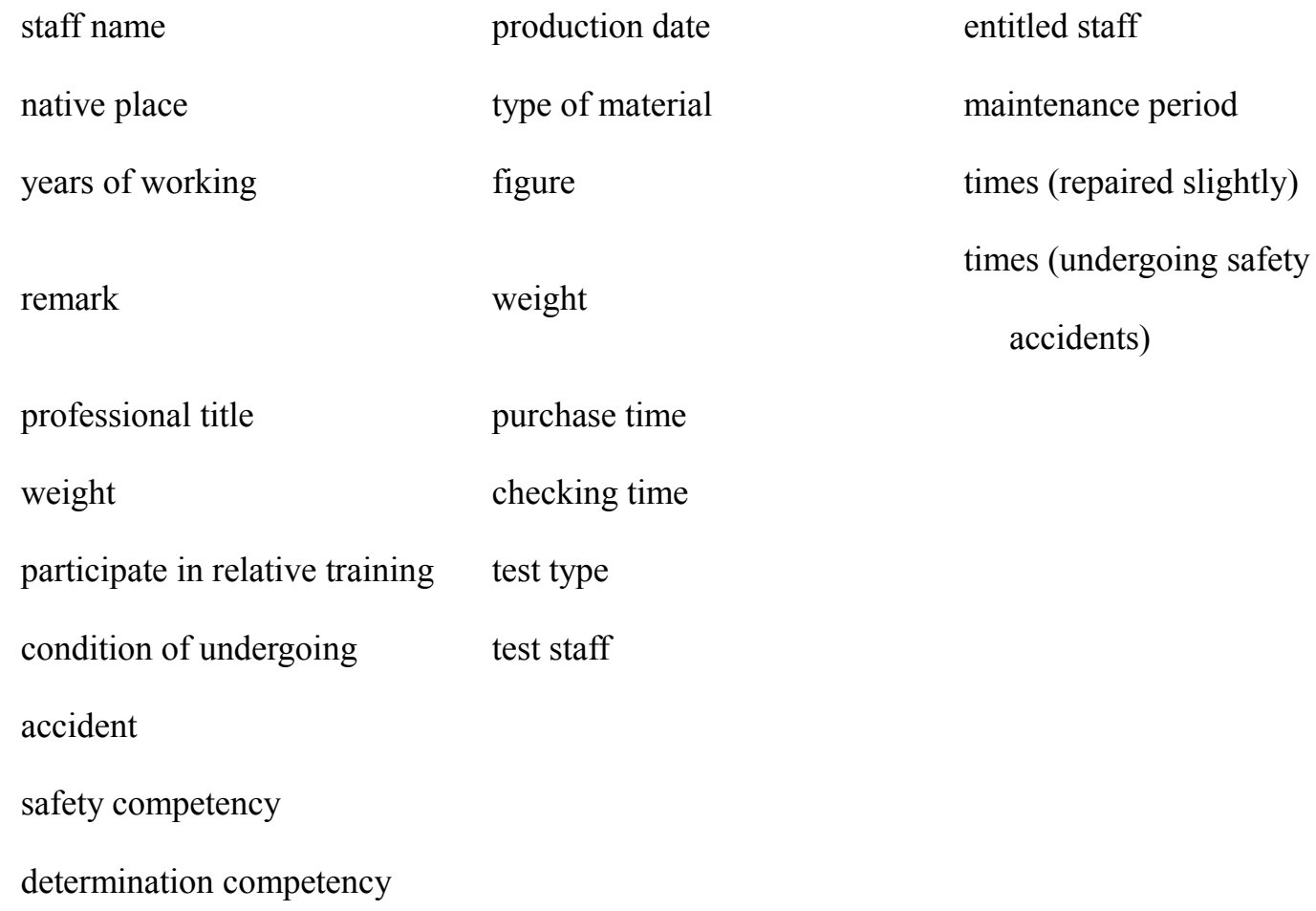

\subsubsection{Tables in ISiCSS}

Tables are the most important components in a database. One of the rules for designing tables is the information classification principle, which indicates that one table is only pertinent to one subject and there should not be repetitious information among tables. Another principle is normal forms (NF) principle (Codd, 1970; Fagin, 1981; Kent, 1983). Based on these principles, 15 tables have been designed in ISiCSS, which are classified into three types of information concerning on-site staff, material and machine. These information types are illustrated in Table 3, Table 4 and Table 5 and the relationships among 15 tables are depicted in Fig. 3.

Table 3. Field Information of On-site staff.

\begin{tabular}{cccccc}
\hline Field Name & Staff ID & Staff Name & Nationality & Native Place & Sex \\
Data Type & text & text & text & text & text \\
\hline Field Name & Stature & Weight & Telephone & Type of Staff & Remark \\
Data Type & numeric & numeric & text & text & memo \\
\hline \multirow{2}{*}{ Field Name } & Educational & Type of & Professional & & \\
& Background & Company & Title & & \\
\hline
\end{tabular}




\begin{tabular}{|c|c|c|c|}
\hline Data Type & text & text & text \\
\hline
\end{tabular}

Table 4. Field Information of Material.

\begin{tabular}{ccccc}
\hline $\begin{array}{c}\text { Field Name } \\
\text { Data Type }\end{array}$ & Material ID & Material Name & Purchase Time & Production Date \\
text & text & date/time & date/time \\
\hline Field Name & Type of Material & By Piece & Figure & Volume \\
Data Type & text & yes/no & text & numeric \\
\hline Field Name & Material & Production & Checker & Checking time \\
Darchaser & Company & text & date/time \\
\hline Field Name & Expiration Date & Weight & Remark & \\
Data Type & date/time & numeric & memo & \\
\hline
\end{tabular}

Table 5. Field Information of Machine.

\begin{tabular}{cccc}
\hline Field Name & Equipment ID & Equipment Name & Production Company \\
Data Type & text & text & text \\
\hline Field Name & Using Time & Remark & Entitled Staff \\
Data Type & numeric & memo & text \\
\hline Field Name & Production Date & Expiration Date & Type of Equipment \\
Data Type & date/time & date/time & text \\
\hline
\end{tabular}




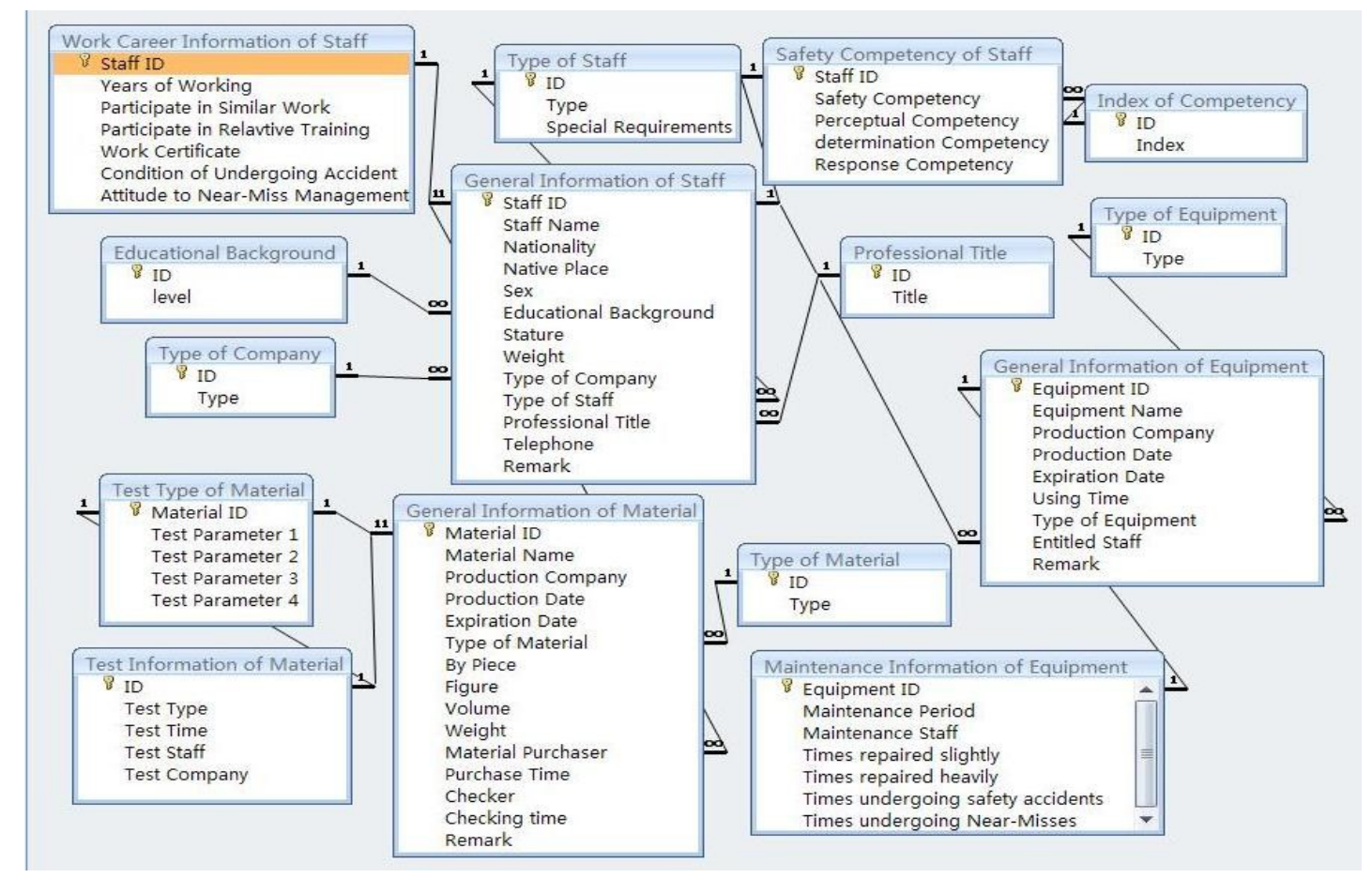

Fig. 3. Relationships among Tables.

\subsubsection{Query in ISiCSS}

Query is a convenient tool to obtain special data on tables. According to the conditions given, required records of tables can be found quickly by query. These records can then constitute a new table for further processing. In ISiCSS, 54 queries are designed according to different information in construction site safety.

\subsubsection{Report in ISiCSS}

Report is an effective way to highlight data from database in a pre-defined format of print. Based on the 54 queries, corresponding reports can be made. Furthermore, reports of complete records of 15 tables can also be intercalated.

\subsubsection{Form, Macro and Module in ISiCSS}

Form plays an important role as a link between database and users. Users are able to input, search and maintain data conveniently using forms. Based on tables, queries and reports in ISiCSS, 10 forms are designed. Moreover, corresponding Macros and VBA codes are edited, in order to make optional group controls and commands effective. Fig. 4 illustrates the main form in ISiCSS. 


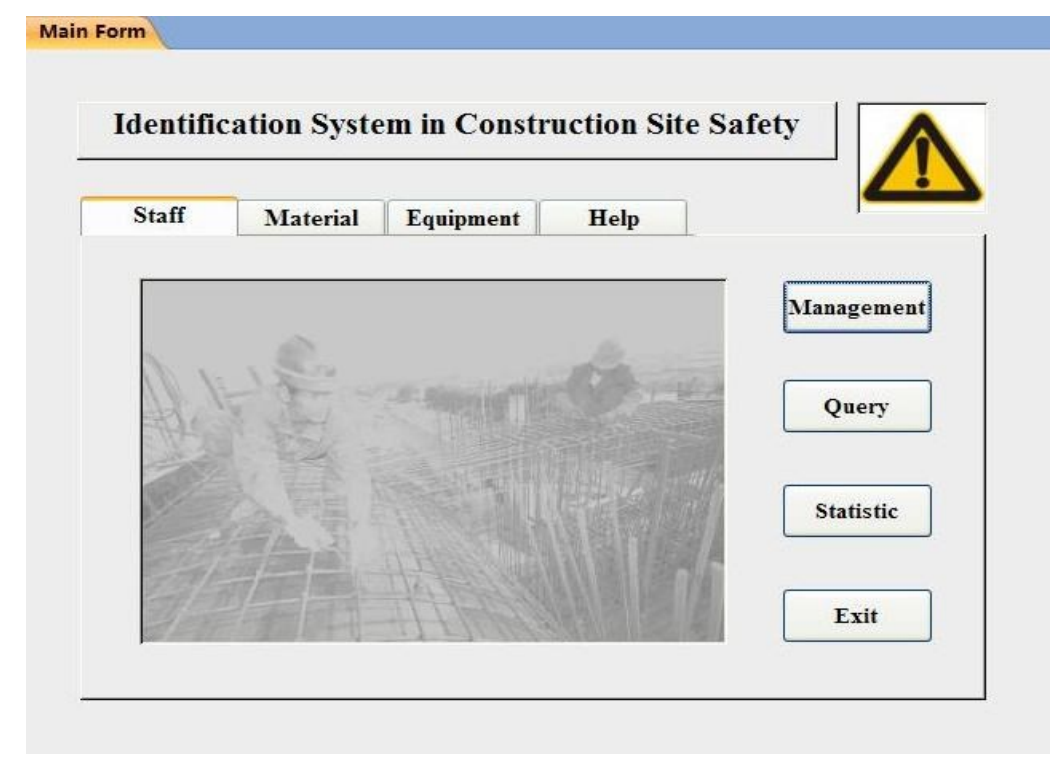

Fig. 4. The main form in ISiCSS.

\subsection{Demonstration system}

\subsubsection{System implementation illustration in construction site}

Fig. 5 describes how the proposed system architecture can be implemented in a construction site. Router devices are deployed in order to provide a full ZigBee network coverage in the construction site. There are three primary types of tagged objects: the construction equipment/vehicles, workers and materials. UHF passive tags should be deployed on the side of the equipment so that they can be read correctly while entering the field. In our test with UHF passive systems we have found that deploying tags on general security cards, which are worn and displayed either over the chest or at the waistline, may result in missed reading because human body acts as obstacle in UHF frequency band and blocks the read-tag communication at certain directions. Thus for the workers the tags can be attached to the safety helmets that are currently mandatory for staff in construction sites. With the fast-expanding RFID deployment in logistics applications, some of the construction materials can be expected to come with their own UHF tags attached by their manufacturers. For the materials that do not come with their own tag, a tag can also be attached. The tag packaging and the attachment location depend on the type of material and its packaging. Hand-held readers may be required in order to read the tags attached under certain circumstances when the fixed reader's antennas are not able to reach the tags. 


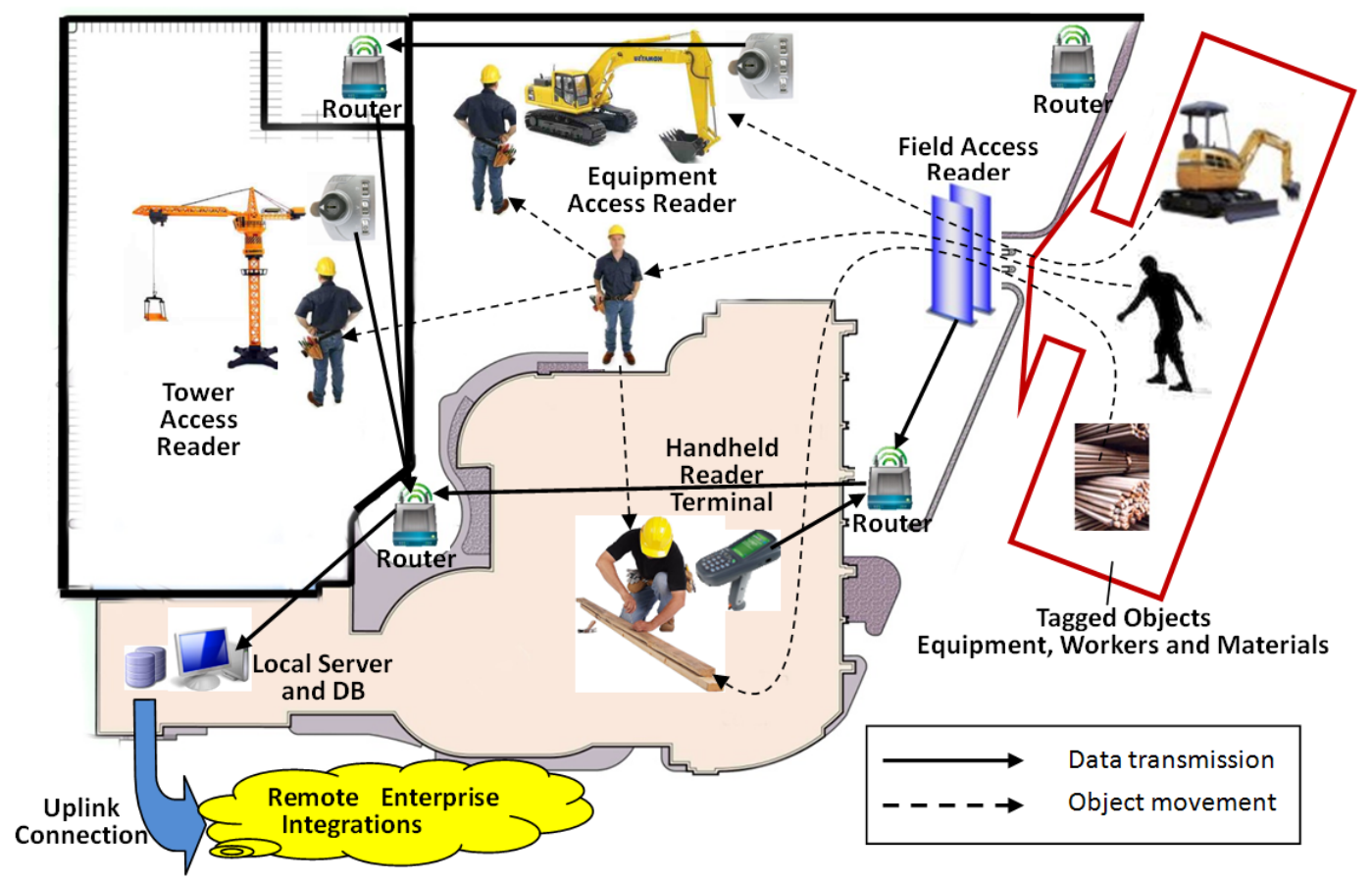

Fig. 5. System implementation illustration in construction site

After entering the site the staff can be equipped with mobile hand-held reader terminals, while the equipment will come with access control readers. When a worker tries to activate a construction equipment, the access control reader carried by the equipment reads the tag attached on the worker's safety helmet. This ID read is then sent back to the local server for approval. If the worker is an authorized person for operating such equipment, the server will return a positive answer with the equipment activation granted. Otherwise, the equipment access will be denied. This implementation requires integration of RFID reader module with equipment's electronic lock or the management system onboard. The mobile hand-held reader terminals are carried by the workers and read the tags attached to the equipment and materials. The tag IDs read are sent back to the server with corresponding information returned depending on the type of enquiry.

The server maintains a database in which the detail information of the available workers, equipment and materials is stored. The reading information reported back from various reader nodes in the system is also logged. This information can be used as record or used by the server's safety monitoring system, which is loaded with predefined safety policies, to generate alarms when necessary. The alarm can be sent to the corresponding nodes located in both router layer and end device layer. A piezoelectric ceramic or a LED light on the nodes board can then be triggered 
to caution the corresponding personnel.

\subsubsection{Demonstration system}

A demonstration system based on our system design is developed as a proof of concept prototype. The demonstration system network backbone is constructed using Jennic JN5139 development kit (Jennic Ltd., 2006), as shown in Fig. 6(a). A Zigbee coordinator establishes the Zigbee network first; several Zigbee routers could then join the network. Thereafter the sink node and various reader nodes can join the network on a plug-and-play basis.

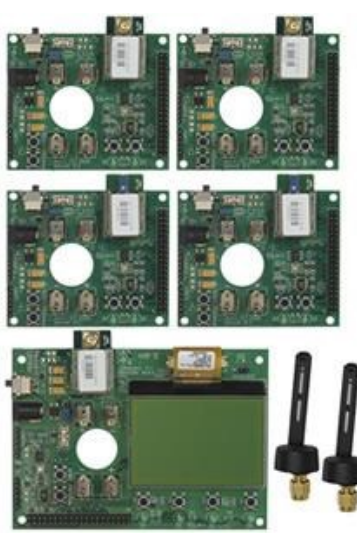

(a)

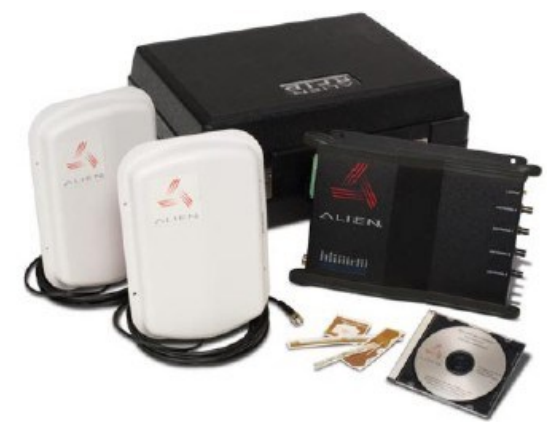

(b)

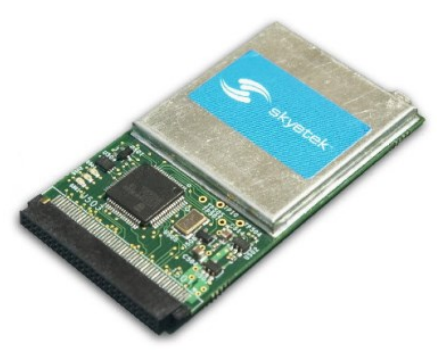

(c)

Fig. 6. (a) Jennic JN5139 kit, (b) Alien ALR-8800 and (c) Skyetek DKM9 module.

Local server and sink node: Using a USB to RS-232 3.3V converter chip, the local server computer can be connected to the UART0 port of a JN5139 ZigBee module. Through the module, which acts as a router in the network, the local server is able to access and retrieve the information it requires from all the other nodes in the network

Reader nodes: The passive tags we used are the typical EPC GEN2 UHF passive RFID tags. The passive reader node is designed by integrating UHF EPC complied reader modules with ZigBee wireless controller modules. In our demonstration system, an Alien ALR-8800 UHF passive reader, which is shown in Fig. 6(b), is chosen to be integrated with a ZigBee end device based on Jennic JN5139 module to construct the high-power reader node for field access control point. A Skyetek DKM9 UHF passive RFID reader embedded module shown in Fig. 6(c) is integrated with a Jennic JN5139 module as the design of low-power reader nodes, which can be 
used either as vehicle access control reader or as hand-held reader.

Laboratory and Field tests: The demonstration system is tested in both a laboratory environment and a small scale field trial with one coordinator, two routers, one sink node with server computer, one high-power RFID reader node and two low-power RFID reader node. Each of the two DKM9 low-power modules is directly integrated with a JN5139 module, with one of them operating as the equipment access reader node and the other as a hand-held reader terminal. We simplified the development process of the high-power reader node by integrating ALR-8800 high-power module with JN5139 module via a small laptop. The laptop is programmed to communicate with both modules as an agent device. This method is easier to be implemented because the reader manufacturer provides reader communication APIs for high level development environment. Since such integration method does not affect the design of our system structure, we consider it sufficient for concept proof in the demonstration system.

The initial test was carried out in Loughborough University's network and control laboratory. The system operates smoothly in the laboratory test with all the designed functions working correctly. A further field test was carried out in a local industry park, where the environment is considered close to a construction site containing both multi-storey structure and open area. As shown in Fig. 7, the high-power reader node is positioned at the road entrance of a sub-block containing heterogeneous structure types. The UHF passive RFID tag is attached on a worker's safety helmet as well as on the side of a forklift. When the worker or the forklift passed the entrance the high-power reader node was able to identify and transmit their IDs back to the server. The passive tags are also attached on some sample materials, and with the low-power reader node the worker is able to read, display and transmit the material ID back to the server. When the low-power reader node is installed on the forklift and the worker in the driving seat, it can read out the worker's ID and send back to server for access granting. The server returns the decision of whether to grant the access, which is displayed on the screen of the low-power reader node. The demonstration system operated correctly and reliably in the field test, which proved the feasibility of our design. Consequently, further analysis can be conducted in the information database based on the primary key information of IDs. 


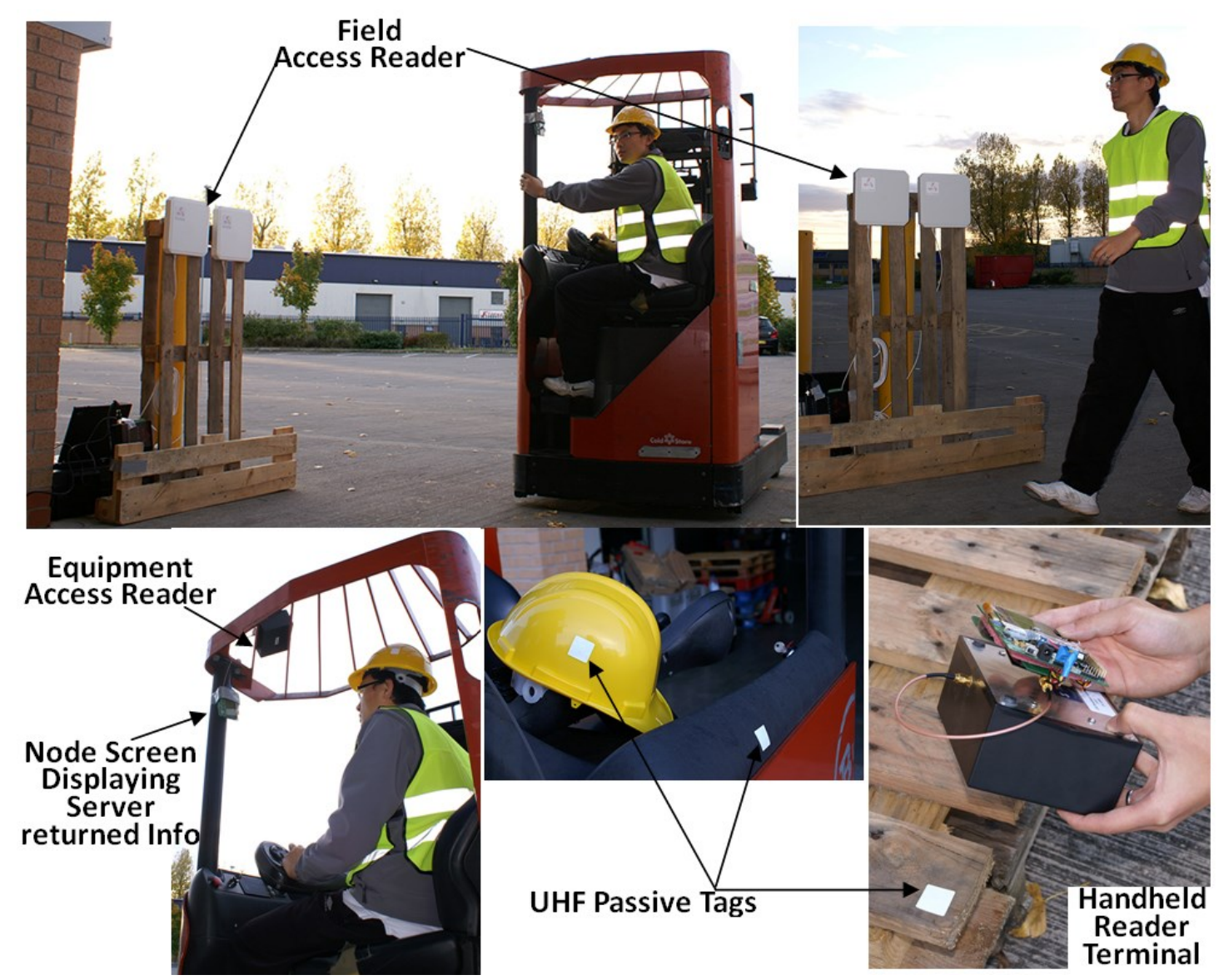

Fig. 7. Demonstration system field test.

\section{Discussion and Conclusion}

Accidents are just the tip of an iceberg. Near-miss accidents were usually referred to as precursors of accidents. Cambraia et al. (2010) suggested that the proactive nature of a near-miss accident was linked to the fact that the items of information generated allow actions to be performed, which would prevent injury or damage to property from occurring in the future. Thus, we argued that precursors and near-miss accidents would provide insight into accidents that could happen and have the great significance to further improve safety margins from the perspective of real-time tracking. While the fatality and injury records in construction continue to plague the industry, many organizations had attempted to develop programs to identify and benefit from accident precursors information, which has a great potential to improve safety performance on construction sites. Information of identity is a crucial part of precursors that holds great importance for safety management of construction sites. The information provided include staff IDs, photos, access authorities, time of inspection, access control, personnel who conducts the 
checking, conditions of the machines, repair work done, etc. Outcomes of recent research have shown that it is possible to track construction assets based on RFID technology (Chae and Yoshida, 2010; Jang and Skibniewski, 2009; Goodrum et al., 2006;). As an attempt to address the need for more research into both integrated analysis of automatic identification requirement and its technological solutions based on real time information on construction sites, this paper designed and implemented an identification system to improve the construction site safety via a proactive accident prevention process.

A case study was first conducted to analyze the automatic identification requirement in construction site safety. Results have shown that it mainly consists of three aspects, namely access control, training and inspection information and operation authority. The system is then designed to fulfill these requirements based on the ZigBee technology, UHF passive RFID technology and the ZigBee RFID sensor network architecture. Simultaneously, an information database is also designed and completed, which include 15 tables, 54 queries, several reports and forms. At the end, a demonstration system based on the proposed system design was developed as a prove-of-concept prototype. The demonstration system operated as expected with all core functionalities. It shows the feasibility of our system and hardware design and their ability of tracking the near-miss events in the scenario of construction site. There are two minor functions described in our system structure design which were not involved in the demonstration prototype. The first minor function is the integration of low-power RFID reader node with the electronic lock or the management system onboard the construction equipment, which is the forklift in our case, as this is not possible at the current stage of our research unless an equipment manufacturer is involved. However, we have shown that the worker's ID can be retrieved by the low-power reader nodes and verified on the server, which demonstrates the feasibility of implementing equipment access control with the design of our low-power reader node. The other minor function not shown in the demonstration system is the "up-link" connection from the local server to a remote center, which was mentioned at the end of Section 3.2.2, as it was considered to be independent of the architecture design of the on-site system and would require expensive pieces of equipment to demonstrate such a link.

In summary, this paper investigated the key technical requirements of tracking near-miss accidents in construction sites and a corresponding system design that could serve as a technical 
reference solution for tracking identity information for proactive accident prevention. Such a study is of great significance and importance for improving safety performance on construction sites. Moreover, as $\mathrm{Wu}$ et al. (2010b) indicated that information of identity, location and environment could also be integrated into one ZigBee enabled RFID sensor network, this study could further serve as a reference design for future system integrations, in which more functions, such as environment monitoring and real-time location tracking, are considered.

\section{Acknowledgements}

The research work for this paper is funded by the National Natural Science Foundation of China (Grant No. 51008073 and 50878049).

\section{Reference}

Aneziris, O.N., Papazoglou, I.A., Baksteen, H., Mud, M., Ale, B.J., Bellamy, L.J., Hale, A.R., Bloemhoff, A., Post, J., Oh, J., 2008. Quantified risk assessment for fall from height. Safety Science 46, 198-220.

Aneziris, O.N., Papazoglou, I.A., Kallianiotis, D., 2010. Occupational risk of tunneling construction. Safety Science, 48(8): 964-972.

Baronti, P., Pillai, P., Chook, V.W.C., Chessa, S., Gotta, A., Hu, Y.F., 2007. Wireless sensor networks: A survey on the state of the art and the 802.15.4 and ZigBee standards. Computer Communications 30(7), 1655-1695

Beavers, J.E., Moore, J. R., Schriver, W.R., 2009. Steel erection fatalities in the construction industry. Journal of Construction Engineering and Management, 135(3), 227-234.

Bier, V. M., Mosleh, A., 1990. The analysis of accident precursors and near misses: implications for risks assessment and risk management. Reliability Engineering and System Safety, 27(1): $91-101$.

Brazier, A. J., 1994. A summary of incident reporting in the process industry. Journal of Loss Prevention in the Process Industries, 7 (3): $243-248$.

Cambraia, F. B., Saurin, T. A., Formoso, C. T., 2010. Identification, analysis and dissemination of information on near misses: A case study in the construction industry. Safety Science, 48(1): 
91-99.

Carter, G., Smith, S.D., 2006. Safety hazard identification on construction projects. Journal of Construction Engineering and Management, 132(2), 197-205.

Chae, S., Yoshida, T., 2010. Application of RFID technology to prevention of collision accident with heavy equipment. Automation in Construction 19, 368-374

Cheng, C. W., Leu, S. S., Lin, C. C., Fan, C., 2010. Characteristic analysis of occupational accidents at small construction enterprises. Safety Science,2010, 48(6):698-707

Chi, C.F., Chang, T.C., Ting, H.I., 2005. Accident patterns and prevention measures for fatal occupational falls in the construction industry. Applied Ergonomics, 36, 391-400.

Chi, C.F., Yang, C.C., Chen, Z.L., 2009. In-depth accident analysis of electrical fatalities in the construction industry. International Journal of Industrial Ergonomics, 39(4): 635-644

Codd, E.F., 1970. A Relational Model of Data for Large Shared Data Banks. Communications of the ACM 13(6), 377-387.

Darbra, R.M., Casal, J., 2004. Historical analysis of accidents in seaports. Safety Science 42, $85-98$

Ergen, E., Akinci, B., 2007. An Overview of Approaches for Utilizing RFID in Construction Industry. Proceedings of RFID Eurasia, 1st Annual, 1-5.

Fagin, R., 1981. A Normal Form for Relational Databases That Is Based on Domains and Keys. Communications of the ACM 6, 387-415.

Gibb, A., Haslam, R., Gyi, D., Hide, S., Duff, R., 2006. What causes accidents? Civil Engineering 159 (2): 46 50.

Goh, Y. M. and Love, P. E.D., 2010. Adequacy of personal fall arrest energy absorbers in relation to heavy workers. Safety Science, 2010, 48(6): 747-754.

Goodrum, P.M., McLaren, M.A., Durfee, A., 2006. The application of active radio frequency identification technology for tool tracking on construction job sites. Automation in Construction $15(3), 292-302$.

Hinze, J., Huang, X.Y., Terry, L., 2005. The nature of struck-by accidents. Journal of Construction Engineering and Management, 131(2), 262-268.

Huang, G.Q., Zhang, Y.F., Jiang, P.Y., 2007. RFID-based wireless manufacturing for walking-worker assembly islands with fixed-position layouts. Robotics and 
Computer-Integrated Manufacturing 23(4), 469-477.

Jang, W. K., Skibniewski, M. J., 2009. Embedded system for construction asset tracking combining radio and ultrasound signals. Journal of Computing in Civil Engineering 23 (4), 221-229.

Jennic Ltd., 2006. JN5139 Module Datasheet. Retrieved Aug 01， 2008， from http://www.jennic.com/files/support_files/JN-DS-JN5139MO-1v4\%20released.pdf

Jones, S., Kirchsteiger, C., Bjerke, W., 1999. The importance of near miss reporting to further improve safety performance. Journal of Loss Prevention in the Process Industries 12 (1): 59-67.

Katz, J.E., Rice, R.E., 2009. Public views of mobile medical devices and services: A US national survey of consumer sentiments towards RFID healthcare technology. International Journal of Medical Informatics 78(2), 104-114.

Kelepouris, T., Pramatari, K., Doukidis, G., 2007. RFID-enabled traceability in the food supply chain. Industrial Management \& Data Systems 107(2), 183-200.

Kent, W., 1983. A Simple Guide to Five Normal Forms in Relational Database Theory. Communications of the ACM, 26, 120-125.

Li, M., Liu, Y., 2007. Underground structure monitoring with wireless sensor networks. Proceedings of conference on Information processing in sensor networks: 69-78.

Liang, N.C., Chen, P.C., Sun, T., Yang, G., Chen, L.J., Gerla, M., 2006. Impact of Node Heterogeneity in Zigbee Mesh Network Routing. IEEE International Conference on Systems, Man and Cybernetics (SMC06), 1, 187-191

Lu, W., Huang, G.Q., Li, H., 2010. Scenarios for applying RFID technology in construction project management. Automation in Construction, doi:10.1016/j.autcon.2010.09.007

Manu, P., Ankrah, N., Proverbs, D., Suresh, S., 2010. An approach for determining the extent of contribution of construction project features to accident causation. Safety Science 48, $687-$ 692

Navon, R., Berkovich, O., 2005. Development and on-site evaluation of an automated materials management and control model, Journal of Construction Engineering and Management, 131(12): 1328-1336.

Navon, R., Sacks, R., 2007. Assessing research issues in Automated Project Performance Control (APPC). Automation in Construction, 16: $474-484$. 
Paek, J., Chintalapudi, K., Govindan, R., Caffrey, J., Masri, S., 2005. A wireless sensor network for structural health monitoring: performance and experience. Proceedings of Embedded Networked Sensors (EmNetS-II): 1-10.

Phimister, J.R., Bier, V.M., Kunreuther (Eds), H.C., 2004. Accident precursor analysis and management: reducing technological risk through diligence. National Academy Press, Washington, DC.

Saurin, T.A., Guimaraes, L.B.D.M., 2008. Ergonomic assessment of suspended scaffolds. International Journal of Industrial Ergonomics, 38, 238 - 246.

Sazonov, E., 2004. Wireless intelligent sensor network for autonomous structural health monitoring. Proceedings of the Society of Photo-optical Instrumentation Engineers (SPIE), 5384: 305-314.

Sun, T., Liang, N.C., Chen, L.J., Chen, P.C., and Gerla, M., 2007. Evaluating Mobility Support in Zigbee Networks. IFIP International Conference on Embedded and Ubiquitous Computing, Southampton, UK

Suraji, A., Duff, A.R., 2001. Identifying root causes of construction accidents: comments. Journal of Construction Engineering and Management, 4, 348-349.

Teizer, J., Allread, B. S., Mantripragada, U., 2010a. Automating the blind spot measurement of construction equipment. Automation in Construction, 19: 491-501.

Teizer, J., Allread, B. S., Fullerton, C. E., Hinze, J., 2010b. Autonomous pro-active real-time construction worker and equipment operator proximity safety alert system. Automation in Construction, 19: 630-640.

Wang, L.C., 2008. Enhancing construction quality inspection and management using RFID technology. Automation in Construction 17, 467-479.

Wang, L.C., Lin, Y.C., Lin, P.H., 2007. Dynamic mobile RFID-based supply chain control and management system in construction. Advanced Engineering Informatics 21, 377-390.

Wu, W., Gibb, A., Li, Q., 2010a. Accident precursors and near misses on construction sites: An investigative tool to derive information from accident databases. Safety Science 48(7), 845-858.

Wu, W., Yang, H., Chew, D., Yang, S., Gibb, A., Li, Q., 2010b. Towards an autonomous real-time tracking system of near-miss accidents on construction sites. Automation in Construction 19(2), 
134-141.

Yang, H., and Yang, S.H., 2007. RFID Sensor Network - Network Architectures to Integrate RFID, Sensor and WSN. Measurement and Control 40(2), 56-59. 